\title{
Improved Outcome after Peripheral Nerve Injury in Mice with Increased Levels of Endogenous Omega-3 Polyunsaturated Fatty Acids
}

\author{
Stacy J. Gladman, ${ }^{1 \star}$ Wenlong Huang, ${ }^{1 \star}$ Siew-Na Lim, ${ }^{1,5}$ Simon C. Dyall, ${ }^{1,4}$ Sophie Boddy, ${ }^{1}$ Jing X. Kang, ${ }^{2}$ \\ Martin M. Knight, ${ }^{3}$ John V. Priestley, ${ }^{1}$ and Adina T. Michael-Titus ${ }^{1}$ \\ ${ }^{1}$ Centre for Neuroscience and Trauma, Blizard Institute, Barts and the London School of Medicine and Dentistry, Queen Mary University of London, \\ London, E1 2AT, United Kingdom, ${ }^{2}$ Laboratory of Lipid Medicine and Technology, Department of Medicine, Massachusetts General Hospital and Harvard \\ Medical School, Boston, Massachusetts 02114, ${ }^{3}$ Medical Engineering Group, School of Engineering and Material Science, Queen Mary University of London, \\ London, E1 4NS, United Kingdom, ${ }^{4}$ Department of Life Sciences, Roehampton University, Whitelands College, London, SW15 4JD, United Kingdom, and \\ ${ }^{5}$ Department of Neurology, Chang Gung Memorial Hospital and Chang Gung University College of Medicine, Taipei 105, Taiwan
}

Functional recovery after a peripheral nerve injury (PNI) is often poor. There is a need for therapies that protect neurons against injury and enhance regeneration. Omega-3 polyunsaturated fatty acids (PUFAs) have been shown to have therapeutic potential in a variety of neurological disorders, including acute traumatic injury. The objective of this study was to assess the neuroprotective and proregenerative potential of $\omega$-3 PUFAs in PNI. We investigated this in mice that express the fat-1 gene encoding for $\omega$ - 3 fatty acid desaturase, which leads to an increase in endogenous $\omega$-3 PUFAs and a concomitant decrease in $\omega-6$ PUFAs. Dorsal root ganglion (DRG) neurons from wild-type or fat-1 mice were subjected to a mechanical strain or hypoxic injury, and cell death was assessed using ethidium homodimer-1 labeling. The fat-1 background appears to confer robust neuroprotection against both injuries. We then examined the early functional and morphological changes in wild-type and fat-1 mice after a sciatic nerve crush. An accelerated functional recovery $7 \mathrm{~d}$ after injury was seen in fat-1 mice when assessed using von Frey filaments and the sciatic nerve functional index. These observations were also mapped to changes in injury-related markers. The injury-induced expression of ATF- 3 was decreased in the DRG of fat- 1 mice, whereas the axons detected $6 \mathrm{~mm}$ distal to the crush were increased. Fat-1 animals also had some protection against muscle atrophy after injury. In conclusion, both in vitro and in vivo experiments support the idea that a higher endogenous $\omega-3$ PUFA could lead to beneficial effects after a PNI.

\section{Introduction}

Axons in the adult peripheral nervous system are able to regenerate when damaged. However, despite advances in surgical techniques, satisfactory recovery usually occurs only in minor nerve injuries or when the distance over which regeneration must occur is small (Jaquet et al., 2001). Functional disability after a peripheral nerve injury (PNI) can have a profound effect on the patient's life and a large socioeconomic impact (Rosberg et al., 2005). Hence, there is a need for new therapies that protect injured

Received July 3, 2011; revised Oct. 25, 2011; accepted Nov. 7, 2011.

Author contributions: S.J.G., W.H., M.M.K., J.V.P., and A.T.M.-T. designed research; S.J.G., W.H., S.-N.L., S.C.D., and S.B. performed research; J.X.K. contributed unpublished reagents/analytic tools; S.J.G. analyzed data; S.J.G. wrote the paper.

We gratefully acknowledge support through a Biotechnology and Biological Sciences Research Council doctoral training grant PhD studentship (S.J.G.) and a Medical Research Council/Engineering and Physical Sciences Research Council Discipline Bridging Initiative award.

*S.J.G. and W.H. contributed equally to this work.

Correspondence should be addressed to Stacy Gladman, Centre for Neuroscience and Trauma, Blizard Institute, Barts and the London School of Medicine and Dentistry, Queen Mary University of London, London, E1 2AT, UK. E-mail:s.gladman@qmul.ac.uk.

DOI:10.1523/JNEUROSCI.3371-11.2012

Copyright $\odot 2012$ the authors $\quad 0270-6474 / 12 / 320563-09 \$ 15.00 / 0$ peripheral neurons and enhance regeneration, thus improving functional outcome in patients.

Polyunsaturated fatty acids (PUFAs) are essential compounds that can be obtained through the diet. Long-chain $\omega$-3 PUFAs, such as docosahexaenoic acid (DHA), have been shown to have therapeutic potential in a variety of neurodegenerative conditions (for review, see Dyall and Michael-Titus, 2008). Our group has also shown the neuroprotective effect of DHA in models of spinal cord injury (SCI) (King et al., 2006; Huang et al., 2007; Ward et al., 2010). One aspect concerning the use of $\omega-3$ PUFAs in neurotrauma that remains to be fully explored is their potential neuroprotective and pro-regenerative effect after PNI. However, $\omega-3$ PUFAs can enhance neurite outgrowth in cultures of hippocampal and cortical neurons (Calderon and Kim, 2004), as well as in cultures of primary sensory neurons (Robson et al., 2010), and DHA has been shown to increase the number of sprouting fibers after a facial nerve transection (Makwana et al., 2006).

For many years, humans evolved on a diet with an $\omega-6 / \omega-3$ ratio of 1-2:1, whereas the present ratio in Western diets is 15$20: 1$. This change is attributable to an increase in the intake of $\omega-6$ PUFAs and a concomitant decrease in the intake of $\omega$-3 PUFAs 
(Simopoulos, 2006). This leads to a deficiency in $\omega-3$ PUFAs, which is a known risk factor in many diseases (Simopoulos, 2008), and could also have a negative impact after a nerve injury. The aim of this study is to assess the effect of increasing tissue levels of $\omega$-3 PUFA on the response to a PNI. Such an increase could be achieved through dietary supplementation or alternatively through the use of the recently developed fat-1 mouse (Kang et al., 2004). These mice express the fat-1 gene from Caenorhabditis elegans, which encodes a fatty acid desaturase not normally present in mammals. This enzyme can convert $\omega-6$ into $\omega$-3 PUFAs, leading to enrichment in tissue $\omega$-3 PUFA levels. The use of this model allows us to produce two different tissue fatty acid profiles (i.e., high vs low $\omega-6 / \omega-3$ ratio).

PNI includes elements of mechanical stretch and hypoxia and can induce substantial alterations in sensory neurons. Thus, in the first part of our study, we isolated dorsal root ganglion (DRG) neurons from fat-1 mice to investigate the effect of increasing endogenous $\omega$-3 PUFA on (1) neurite outgrowth and (2) neuronal survival after injury. Subsequently, we examined the early functional and morphological changes induced by $\omega$-3 PUFA in fat-1 mice after a sciatic nerve crush.

\section{Materials and Methods}

Animal care and experimental groups. fat- 1 breeders on a C57BL/6 background were obtained from Dr. Jing Kang (Harvard Medical School, Boston, MA) (Kang et al., 2004), and experimental mice were obtained by mating male fat-1 mice with female C57BL/6 wild-type (WT) mice (Charles River). Mice were housed four to five per cage in a temperaturecontrolled and enriched environment and were maintained on a 14/10 h light/dark cycle with ad libitum access to food and water. All procedures described below were performed during the light cycle. Both the fat-1 transgenic and WT mice were maintained on a diet rich in $\omega-6$ PUFAs (mainly linoleic acid) with very little $\omega$-3 PUFAs $(\sim 0.1 \%$ of the total fat supplied) (TestDiet). WT mice on this supplemented diet were described as "WT $\omega-6$ " mice (mice with low levels of $\omega$-3 PUFAs in tissues), whereas fat- 1 transgenic mice had a higher amount of $\omega$-3 PUFAs but low $\omega-6$ PUFAs in their tissues. For in vitro studies, a third group of mice were used. These were WT mice (Charles River) fed on a standard balanced diet, not supplemented with PUFAs. The study was conducted in accordance with the United Kingdom Animals (Scientific Procedures) Act 1986 and with international guidelines on the ethical use of animals.

Lipid analysis. Spinal cords and DRG were harvested from animals, frozen on dry ice, and stored at $-80^{\circ} \mathrm{C}$ until analysis. Lipids were extracted from spinal cord $(n=4)$ and DRG $(n=3)$ samples using the method of Folch et al. (1957) with $0.01 \%$ w/v 2,6-di-tert-butyl-p-cresol (butylated hydroxytoluene) added as an antioxidant. Total phospholipids were then isolated by thin-layer chromatography (Manku et al., 1983), and the fatty acid composition was measured after transesterification with $14 \%$ borontrifluoride in methanol (Morrison and Smith, 1964) at $100^{\circ} \mathrm{C}$ for $20 \mathrm{~min}$. Individual fatty acids were identified by gas chromatography coupled to mass spectrometry (Agilent 6890 gas chromatograph connected to an Agilent 5973 mass selective detector; Agilent Technologies) using a supelcowax 10 capillary column $(30 \mathrm{~m} \times$ $0.25 \mathrm{~mm} \times 0.25 \mathrm{~mm}$; Sigma), as described previously (Dyall et al., 2007). Lipid identity was confirmed by retention times compared with known standards and mass spectra comparison with the National Institute of Standards and Technology database. Quantification was performed on selected ion peak area by ChemStation software (Agilent Technologies). Corrections were made for variations in the detector response, and values of detected fatty acids were normalized to $100 \%$ and expressed as moles percentage (mol\%).

Primary DRG neuron culture. Primary neuronal cultures were prepared as described previously (Gladman et al., 2010). Cervical to lumbar level DRG were harvested from adult C57BL/6 WT or fat-1 mice aged 2-3 months. DRGs were dissociated chemically with collagenase $(0.125 \%$ and then raised to $0.25 \%$ ) (Type 4; Worthington Biochemicals), followed by $0.25 \%$ trypsin (Sigma). The tissue was then triturated with a pipette tip until homogeneously dissociated. The cell suspension was centrifuged and resuspended in serum-free medium (BSF-2) consisting of $1 \% \mathrm{~N}-2$ supplement (Invitrogen), $0.3 \%$ bovine serum albumin (Fraction V; Sigma), and $100 \mathrm{U} / \mathrm{ml}$ penicillin per $100 \mu \mathrm{g} / \mathrm{ml}$ streptomycin (Sigma) in Ham's F-12 medium (Invitrogen). Cells were plated out onto either glass coverslips or elastic membranes (Dunlab) double-coated with poly-Dlysine ( $5 \mu \mathrm{g} / \mathrm{ml}$; Sigma) and laminin (10 $\mu \mathrm{g} / \mathrm{ml}$; Sigma). Cultures were maintained at $37^{\circ} \mathrm{C}$ in a humidified atmosphere with $5 \% \mathrm{CO}_{2} / 95 \%$ air for $48 \mathrm{~h}$ before additional experiments.

In vitro injury models. The methods used were as described by Gladman et al. (2010). Briefly, for the mechanical injury, a Flexcell Tension Plus FX-4000T system (Flexcell International) was used. DRG neurons were grown on precoated elastic membranes within BioFlex six-well culture plates (Dunlab) for $48 \mathrm{~h}$ in BSF-2 medium. A $20 \%$ tensile strain was applied to each membrane for $1 \mathrm{~h}$, followed by a $24 \mathrm{~h}$ unstrained period. The strain was applied and removed at a controlled strain rate of $2 \% / \mathrm{s}$. For unloaded controls, Bioflex plates seeded with DRG neurons were maintained in an identical manner but in the absence of any membrane distortion. All cultures were maintained at $37^{\circ} \mathrm{C}$ in a humidified atmosphere with $5 \% \mathrm{CO}_{2} / 95 \%$ air throughout the course of the experiment. For the hypoxic injury DRG neurons were cultured on laminin-coated glass coverslips in BSF-2 for $48 \mathrm{~h}$ before being subjected to hypoxic conditions of $2 \%$ oxygen $\left(5 \% \mathrm{CO}_{2}, 93 \% \mathrm{~N}_{2}\right)$ for $24 \mathrm{~h}$. The BSF- 2 medium was acclimatized in the low-oxygen conditions for $24 \mathrm{~h}$ before adding to the cultures at the start of the hypoxic period. Control cultures were maintained at $18 \%$ oxygen concentration throughout.

Ethidium homodimer-1 assessment of cell death. Injury to cultured DRG neurons was assessed by measuring uptake of ethidium homodimer-1 (EthD-1) (Invitrogen), a cell-impermeable DNA fluorochrome that is rapidly taken up into the nuclei of cells with compromised membranes. At the end of the injury period and corresponding control periods, cultures were incubated with $1 \mu \mathrm{M} \mathrm{EthD-1}$ in F-12 medium for 15 min at $37^{\circ} \mathrm{C}$ and then washed and fixed with $4 \%$ paraformaldehyde (PFA). They were immunostained with the primary antibody for the pan-neuronal marker rabbit anti-protein gene product (PGP) 9.5 (1:1000; Sigma). Cultures were washed with PBS and then incubated with the secondary antibody anti-rabbit Alexa Fluor-488 (1:2000) and the fluorescent nuclear dye bisbenzimide (Hoechst 33342) ( $2 \mu \mathrm{g} / \mathrm{ml}$; Sigma). Cells were observed at $20 \times$ magnification using a Leica DMRD microscope. The percentage of neuronal cell death was determined in six randomly chosen fields of view from three separate culture wells (18 fields in total) for each animal $(n=3)$ in each group.

Basal neurite growth assay. L4 and L5 DRG neurons were dissected from WT $\omega-6$ and fat -1 mice and cultured for $18 \mathrm{~h}$. Cells were then fixed in 4\% PFA for 5 min before being incubated overnight at room temperature with PGP 9.5 (1:1000). The slides were washed with PBS before incubation with the secondary antibody goat anti-rabbit biotin (1:1000) for $1.5 \mathrm{~h}$. Slides were washed in PBS and then directly incubated in ABC reagent (1:200; Vector Laboratories) for $30 \mathrm{~min}$. Slides were then washed in PBS before development in a 3-3' -diaminobenzidine solution containing $0.01 \% \mathrm{H}_{2} \mathrm{O}_{2}$ and $0.25 \%$ nickel sulfate. Images of 100 neurons were recorded for each animal $(n=3)$ and imported into MetaMorph analysis program (Molecular Devices). The length of the longest neurite and number of branches were measured for each neuron.

Sciatic nerve crush procedure. For inducing the crush injury, mice were anesthetized by halothane inhalation (1-1.5\%), and the skin was shaved. Under aseptic conditions, a small incision was made on the skin at the mid-thigh level, followed by a small incision between the gluteus superficialis and biceps femoris muscles to expose the left sciatic nerve. The nerve was carefully separated from surrounding connective tissue and then crushed by a $15 \mathrm{~s}$ compression with a pair of fine Dumon \# 5 forceps. The animals were monitored for $7 \mathrm{~d}$ after the injury. Both male (eight WT and eight fat-1 positive) and female progeny (three WT and three fat-1 positive) were used in this study, resulting in 11 mice in each group.

Mechanical threshold sensory test. Two baseline measurements of hindpaw reflex sensitivity to punctate static mechanical stimuli, using graded von Frey (VF) nylon filaments were performed for five WT $\omega-6$ and five fat-1 mice before surgical manipulation (pre-injury). Clear Plexiglas 
boxes $(23 \times 18 \times 14 \mathrm{~cm})$ with a $0.8 \mathrm{~cm}$ mesh flooring were used so that the VF filaments were applied from underneath the mouse. VF filaments were applied in ascending order to the mid-plantar surface of the hindpaws. The lowest force leading to at least three withdrawals in five trials was defined as the withdrawal threshold to mechanical stimuli. VF tests were performed on days 1, 4, and 7 after injury.

Motor function evaluation. The assessment of motor function recovery was performed by calculating the sciatic functional index (SFI) before injury and at $7 \mathrm{~d}$ after injury (Inserra et al., 1998; Varejão et al., 2001). The hindpaws of six WT $\omega-6$ and six fat- 1 mice were pressed down onto an ink-soaked sponge before being placed in a narrow enclosed runway (50 $\mathrm{cm}$ long) with a white paper floor ( $5 \mathrm{~cm}$ wide). Three measurements were then taken from the resulting footprints: (1) print length (PL), which is the distance from the heel to the third toe, (2) toe spread (TS), which is the distance from the first to the fifth toe, and (3) intermediary toe spread (IT), which is the distance from the second to the fourth toe. All three measurements from the six most clearly inked foot prints per run were taken from the normal (N) and experimental (E) sides, and the SFI was calculated using the following formula:

$$
\begin{aligned}
\mathrm{SFI}=-38.3\left(\frac{\mathrm{EPL}-\mathrm{NPL}}{\mathrm{NPL}}\right)+109.5\left(\frac{\mathrm{ETS}-\mathrm{NTS}}{\mathrm{NTS}}\right) \\
+13.3\left(\frac{\mathrm{EIT}-\mathrm{NIT}}{\mathrm{NIT}}\right)-8.8 .
\end{aligned}
$$

where $\mathrm{E}$ is the injured paw, and $\mathrm{N}$ is the non-injured paw.

Tissue processing and immunohistochemical analysis. The tissue sections for immunohistochemical analysis were prepared as follows. The animals were killed using $\mathrm{CO}_{2}$ inhalation and perfused via the ascending aorta with saline, followed by $4 \%$ PFA in $0.1 \mathrm{~m}$ phosphate buffer. Tissue was then dissected and postfixed for $4 \mathrm{~h}$ before being cryoprotected overnight at $4^{\circ} \mathrm{C}$ in $30 \%$ sucrose in PBS. For the sciatic nerve, $10 \mu \mathrm{m}$ serial transverse sections were obtained with a cryostat $6 \mathrm{~mm}$ distal to the crush site. For the L4 and L5 DRG tissue, $8 \mu \mathrm{m}$ serial sections were obtained. Cryostat sections were blocked in 10\% normal goat serum (Invitrogen) before overnight incubation with the primary antibody at room temperature in a humidified box. The primary antibodies used were anti-rabbit activating transcription factor 3 (ATF-3) (1:200; Santa Cruz Biotechnology), anti-rabbit growth-associated protein $43 \mathrm{kDa}$ (GAP-43) (1:200; Millipore Bioscience Research Reagents), and anti-rabbit neurofilament $200 \mathrm{kDa}$ (NF200) (1:1000; Sigma). Sections were then washed with PBS and incubated with the appropriate secondary antibodies tagged with Alexa Fluor-488 or Alexa Fluor-594 (1:1000; Invitrogen). Sections were counterstained with the fluorescent nuclear dye bisbenzimide (Hoechst 33342 ) ( $2 \mu \mathrm{g} / \mathrm{ml}$; Sigma). For the muscle, wet weights of the perfused ipsilateral and contralateral gastrocnemius muscles were recorded. Histological serial cross-sections $(10 \mu \mathrm{m})$ of the muscle, cut transversely to the muscle main axis, were obtained with a cryostat and stained with hematoxylin and eosin (H\&E) for morphological evaluation.

Image and data analysis. Sections were viewed on a Leica epifluorescence microscope, and images were captured using a Hamamatsu digital camera and HiPic32 imaging software. Figures were prepared using Adobe Photoshop 7. Quantification of the proportion of ATF-3- and GAP-43-expressing DRG neurons was determined by counting the number of immunoreactive and non-immunoreactive neurons with visible nucleus (as determined by Hoechst staining). Analysis was performed by a blinded observer using a $20 \times$ objective, and values were compared with those obtained for DRG harvested from the contralateral side $(n=6$ per group). At least $250 \mathrm{DRG}$ neurons per mouse were counted on randomly chosen sections from L4 and L5. To assess axonal regeneration, images of the stained transverse sections of sciatic nerve were taken at $10 \times$ magnification. The percentage area of sciatic nerve covered by either NF200 or GAP-43 staining was determined for three randomly chosen sections per animal using the Qwin image analysis software (Leica) and compared with the contralateral nerve ( $n=11$ per group). For the muscle, the cross-sectional areas of 200 randomly selected fibers were measured from three randomly chosen sections per animal from both the ipsilateral and contralateral side using NIH ImageJ software ( $n=11$ per group).
Table 1. Fatty acid composition of phospholipids extracted from DRG $(n=3)$ and spinal cord $(n=4)$ from WT mice and mice expressing the fat-1 gene fed a diet

\begin{tabular}{|c|c|c|}
\hline Fatty acid & WT $\omega$-6 diet & fat-1 $\omega$-6 diet \\
\hline \multicolumn{3}{|l|}{ DRG } \\
\hline 14:0 & $0.75 \pm 0.04$ & $0.73 \pm 0.05$ \\
\hline $16: 0$ & $30.65 \pm 0.73$ & $30.91 \pm 0.71$ \\
\hline 18:0 & $34.38 \pm 0.31$ & $34.55 \pm 0.72$ \\
\hline $20: 0$ & $2.58 \pm 0.29$ & $2.44 \pm 0.24$ \\
\hline$\Sigma$ Saturated & $68.37 \pm 1.38$ & $68.64 \pm 1.71$ \\
\hline 18:1 & $11.78 \pm 0.47$ & $11.53 \pm 0.31$ \\
\hline $20: 1$ & $0.37 \pm 0.03$ & $0.37 \pm 0.03$ \\
\hline$\Sigma$ Monounsaturated & $12.15 \pm 0.51$ & $11.90 \pm 0.34$ \\
\hline $18: 2 n-6(L A)$ & $3.71 \pm 0.15$ & $3.60 \pm 0.20$ \\
\hline $20: 4 n-6(A A)$ & $5.53 \pm 0.07$ & $5.09 \pm 0.14^{*}$ \\
\hline 22:4/5n-6 (DTA/DPA) & $4.33 \pm 0.31$ & $2.44 \pm 0.02^{*}$ \\
\hline$\sum(n-6)$ PUFA & $13.56 \pm 0.52$ & $11.13 \pm 0.35$ \\
\hline 20:5n-3 (EPA) & $0.89 \pm 0.06$ & $0.98 \pm 0.02$ \\
\hline $22: 5 n-3(D P A)$ & $0.91 \pm 0.07$ & $1.68 \pm 0.16^{*}$ \\
\hline 22:6n-3 (DHA) & $4.11 \pm 0.32$ & $5.67 \pm 0.14^{*}$ \\
\hline$\sum(n-3)$ PUFA & $6.18 \pm 0.54$ & $8.32 \pm 0.32$ \\
\hline \multicolumn{3}{|l|}{ Spinal cord } \\
\hline $14: 0$ & $0.51 \pm 0.05$ & $0.43 \pm 0.03$ \\
\hline $16: 0$ & $28.84 \pm 0.61$ & $28.43 \pm 0.76$ \\
\hline $18: 0$ & $34.01 \pm 0.42$ & $33.17 \pm 1.00$ \\
\hline $20: 0$ & $3.60 \pm 0.18$ & $3.82 \pm 0.11$ \\
\hline$\sum$ Saturated & $66.97 \pm 0.32$ & $65.85 \pm 0.47$ \\
\hline 18:1 & $7.67 \pm 0.21$ & $7.91 \pm 0.52$ \\
\hline $20: 1$ & $1.73 \pm 0.08$ & $1.84 \pm 0.05$ \\
\hline$\sum$ Monounsaturated & $9.40 \pm 0.14$ & $9.75 \pm 0.29$ \\
\hline 18:2n-6 (LA) & $2.12 \pm 0.05$ & $2.13 \pm 0.10$ \\
\hline $20: 4 n-6(A A)$ & $5.51 \pm 0.22$ & $5.01 \pm 0.15$ \\
\hline $22: 4 n-6(D P A)$ & $4.33 \pm 0.25$ & $3.55 \pm 0.37$ \\
\hline$\sum(n-6)$ PUFA & $11.96 \pm 0.17$ & $10.69 \pm 0.21$ \\
\hline $20: 5 n-3$ (EPA) & $1.11 \pm 0.05$ & $1.09 \pm 0.12$ \\
\hline $22: 5 n-3(D P A)$ & $1.97 \pm 0.05$ & $3.34 \pm 0.28^{*}$ \\
\hline 22:6n-3 (DHA) & $10.56 \pm 0.57$ & $13.36 \pm 0.28^{*}$ \\
\hline$\sum(n-3)$ PUFA & $13.64 \pm 0.25$ & $17.80 \pm 0.16$ \\
\hline
\end{tabular}
enriched in $\omega$-6 PUFA

Data expressed as mol\% of total fatty acids \pm SD $\left({ }^{*} p<0.05\right)$. EPA, Eicosapentaenoic acid; DTA, docosatetraenoic acid; $A A$, arachidonic acid; $L A$, linoleic acid.

Statistical analysis. For the in vitro study, results are expressed as means \pm SEM of three experiments, with each experiment using ganglia pooled from two animals. A two-way ANOVA was used to compare the overall effect of diet and injury on cell death, followed by post hoc multiple pairwise comparisons using Tukey's test, with $p<0.05$ indicating a statistically significant difference. For the sciatic nerve regeneration study, all data were analyzed by one-way ANOVA, followed by post hoc multiple pairwise comparisons using Tukey's test. Statistical significance was established at $p<0.05$. SPSS (IBM) was used to perform statistical analysis.

\section{Results}

\section{Fatty acid profiles in WT and fat-1 mice}

The fatty acid content of phospholipids, extracted from either the spinal cord or the DRG of WT mice and mice expressing the fat- 1 gene and fed a diet enriched with $\omega-6$ PUFAs, were analyzed (Table 1). The spinal cord and DRG had distinct fatty acid profiles, with higher levels of $\omega-3$ PUFAs in the spinal cord. The greatest difference was observed in the DHA content, which measured at $6.61 \mathrm{~mol} \%$ in the spinal cord and only $4.11 \%$ in the DRG. The expression of the fat 1 gene altered the PUFA composition in both tissues tested, with the most pronounced effects in 22 carbon fatty acid content. Mice expressing the fat-1 gene had significantly elevated levels of both DHA (a 29\% change in the spinal cord and a $46 \%$ change in the DRG) and docosapentaenoic acid 

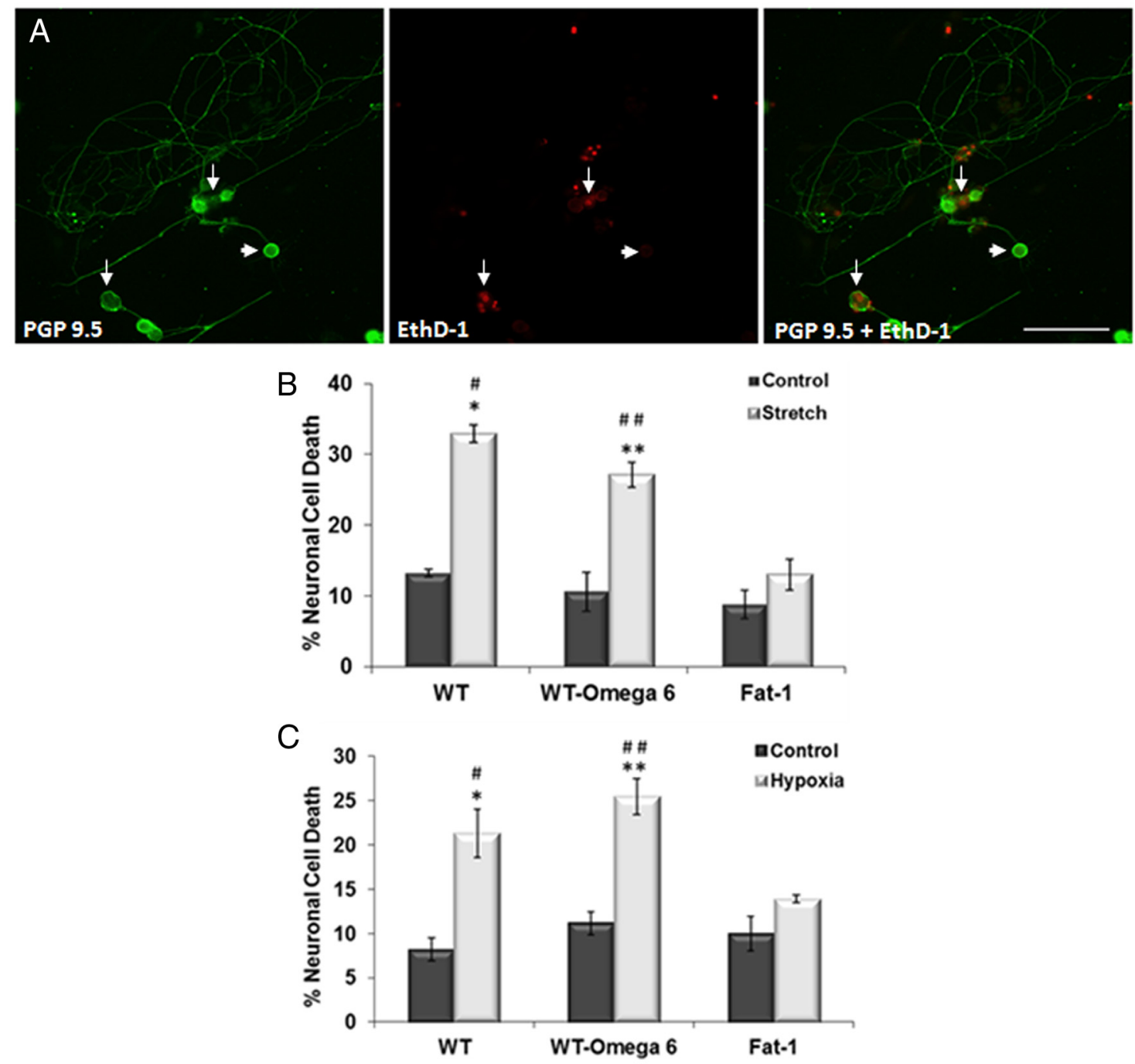

Figure 1. Neuroprotective effect of the fat-1 gene in vitro. DRGs were dissected from WT mice, WT mice fed a diet rich in $\omega-6$, and mice containing the fat- 1 gene fed a high $\omega-6$ diet, and the dissociated cells were cultured for $48 \mathrm{~h}$. $A$, Representative images of DRG cultures showing the method of assessing cell death after injury. EthD- 1 binds to DNA in the nucleus of dead cells and fluoresces red (white arrow indicates dead neuron), whereas live cells have no EthD-1 nuclear stain (arrowhead). Scale bar, $100 \mu \mathrm{m}$. Cultures were exposed to either mechanical stretch injury (B) or hypoxic injury $(\mathbf{C})$, and neuronal cell death assessed with EthD-1. Neuronal injury increased compared with non-injured controls only in the WT and WT $\omega-6$ groups $\left({ }^{*} p<0.05\right.$, $\left.{ }^{* *} p<0.005\right)$. Cell death was significantly reduced in the fat- 1 group compared with both WT and WT $\omega$-6 injured groups ( ${ }^{\#} p<0.05,{ }^{\#} p<0.005$ ). Error bars indicate SEM for $n=3$.

n-3 (DPAn-3) (a 212\% change in the spinal cord and a $257 \%$ change in the DRG). There was also a concomitant decrease in DPAn- 6 ( $-60 \%$ change in the spinal cord and a $-72 \%$ change in the DRG) and docosatetraenoic acid ( $-65 \%$ change in the spinal cord and a $-46 \%$ change in the DRG) in mice expressing the fat-1 gene. Arachidonic acid was also decreased by $13 \%$ in the spinal cord and $7 \%$ in the DRG of fat- 1 mice. There was very little overall change in the total percentage of saturated and monounsaturated fatty acids in either the spinal cord or DRG in fat-1expressing mice.

\section{The effect of the fat-1 gene on the response to injury in vitro}

To determine the effect of changing endogenous fatty acid levels on cell survival after injury, DRG neurons were dissected and cultured from three groups of animals; (1) WT mice, (2) wildtype mice fed on a diet rich in $\omega$-6 (WT $\omega$-6), and (3) mice expressing the fat-1 gene fed on a high $\omega$ - $6 \operatorname{diet}($ fat- 1 ). Figure $1 \mathrm{~A}$ illustrates the method of assessing cell death after injury. EthD-1 was used as the marker of injury, and the cultures were counter- stained with PGP 9.5 to allow the identification of the DRG neurons.

Mechanical injury resulted in a 2.5-fold increase in neuronal cell death compared with unstretched controls in WT cultures (from 13 to $33 \%$ ) and WT $\omega-6$ cultures (from 11 to $27 \%$ ). There was no statistical difference in the response to injury between these two groups. Conversely, the increase in neuronal cell death was almost completely abolished in the cultures from fat-1 mice, with only $13 \%$ of neurons positive for EthD-1 after mechanical stretch. This did not differ significantly from unstretched control fat-1 cultures (9\%) (Fig. 1B). Hypoxia resulted in neuronal cell death increasing significantly from 8 to $21 \%$ in WT cultures (2.6fold increase) and from 11 to $25 \%$ (2.3-fold increase) in WT $\omega$ - 6 cultures. There was no statistical difference in injury response between these two groups. The increase in neuronal cell death was almost completely abolished in the cultures from fat-1 mice, with only $14 \%$ of neurons positive for EthD-1 after hypoxia. This did not differ significantly from normoxic fat-1 cultures (10\%) (Fig. 1C). 

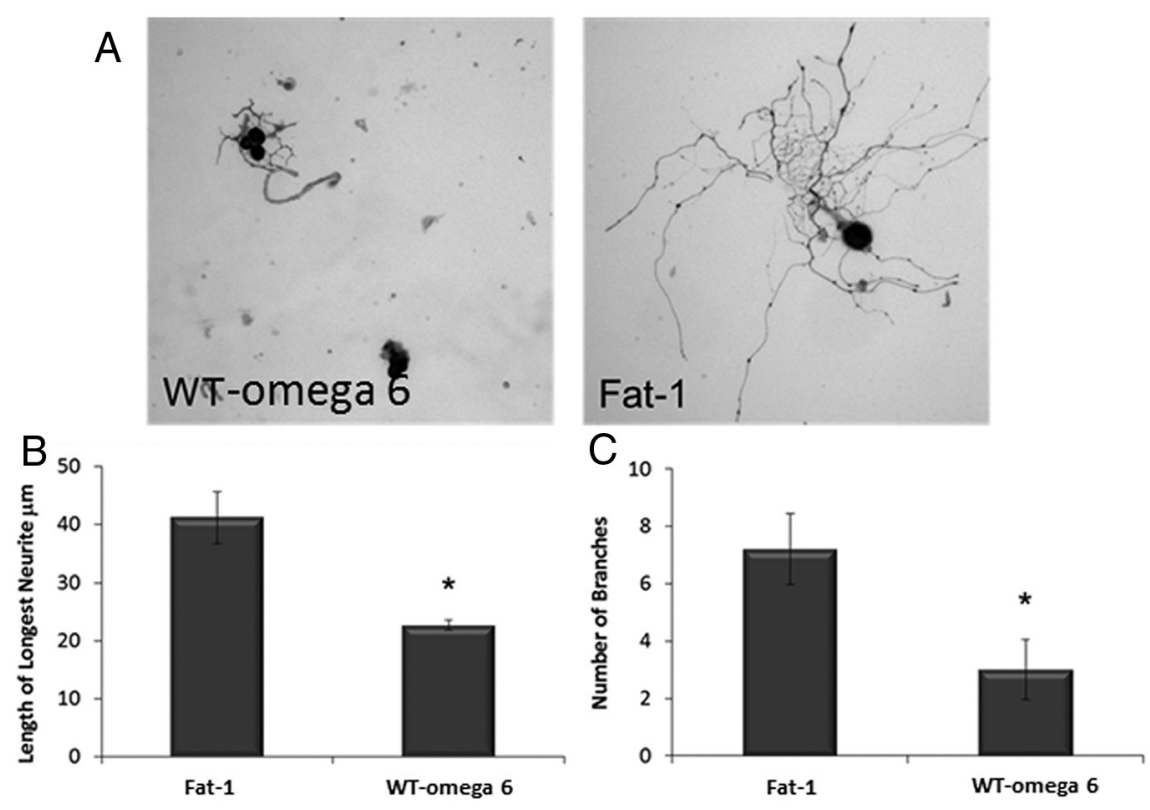

Figure 2. Neurotrophic effects of $\omega-3$ PUFA. A, DRGs were dissected from WT mice or mice containing the fat- 1 gene fed on a high $\omega-6$ diet, and the dissociated cells were cultured for $18 \mathrm{~h}$ before being stained with PGP 9.5 to view neurites. Length of the longest neurite $(\boldsymbol{B})$ and number of branches $(\boldsymbol{C})$ were then assessed. Neurons from fat- 1 mice showed significantly longer neurites and increase in branching than neurons from WT $\omega$-mice $\left.{ }^{*} p<0.05\right)$. Error bars indicate SEM for $n=3$.

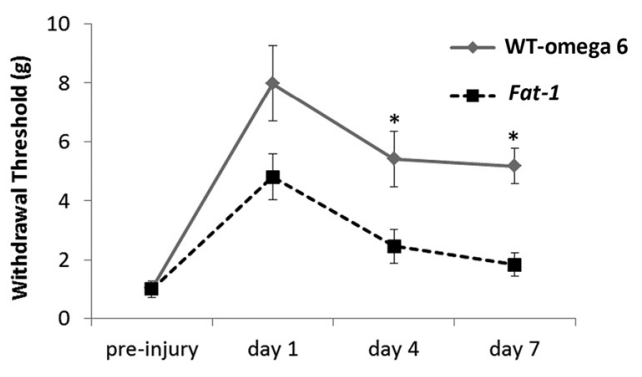

Figure 3. Functional recovery after sciatic nerve crush assessed with VF filaments. The baseline withdrawal threshold of each of the hindpaws using VF filaments as determined for each mouse before surgical manipulation (day 0 ) and on days 1,4 , and $7 \mathrm{~d}$ after injury. There was no difference in pre-injury withdrawal thresholds between WT $\omega$ - 6 and fat- 1 groups, but at $4 \mathrm{~d}$ after injury, there was a statistical difference between the response of the two groups, and this continued to day $7\left({ }^{*} p<0.05\right)$. Error bars indicate SEM for $n=5$ animals per group.

\section{The effect of the fat-1 gene on neurite outgrowth in vitro}

The growth of DRG neurons dissected and cultured from (1) WT mice or (2) mice expressing the fat-1 gene, both groups fed on a high $\omega-6$ diet, was compared. Figure $2 A$ shows representative examples of neurite outgrowth from the two groups. DRG neurons from fat-1 mice showed a more complex neurite outgrowth phenotype, with neurons displaying both longer neurites ( 40 $\mu \mathrm{m}$ compared with $20 \mu \mathrm{m}$ ) and more branches (seven compared with three) than displayed by neurons cultured from WT (Fig. $2 B, C$ ).

\section{The effect of the fat-1 gene on the response to sciatic nerve injury}

The in vitro results obtained suggested that raising the endogenous $\omega-3$ PUFA level had neuroprotective and neuritepromoting effects. We next addressed the question of whether higher tissue $\omega$-3 PUFA has any effect on the recovery after a nerve injury in vivo. A mid-thigh sciatic crush injury was induced in two groups of animals: (1) WT mice fed a diet high in $\omega-6$ (designated WT $\omega-6$ ) and (2) mice expressing the fat-1 gene (designated fat-1).

\section{Functional measures}

Mechanical stimulation thresholds

After a sciatic nerve crush injury, the manual VF test was used to determine the force required to induce a flexion withdrawal response to mechanical stimuli (Fig. 3). No significant difference in baseline response was found between the WT $\omega-6$ and fat- 1 animals, with both groups responding to a $1 \mathrm{~g}$ hair (equivalent to a force of $9.806 \mathrm{mN}$ ). After sciatic nerve crush, the force required to induce a withdrawal response increased in both WT $\omega-6$ and fat- 1 groups, indicating that both groups lose touch sensation. By day 1 after injury, the fat-1 group required on average a $5 \mathrm{~g}$ stimulation to induce a response. This was lower than, although not significantly different from, the $8 \mathrm{~g}$ required by the WT $\omega-6$ group. By day 4 after injury, there was a statistically significant difference in withdrawal threshold between the two groups, with fat-1 mice requiring a $2.5 \mathrm{~g}$ hair to induce a response compared with $5.4 \mathrm{~g}$ in the WT group $(p<0.05)$. Both groups continued to regain sensation up to day 7 , when the recovery response in fat-1 mice remained significantly better than in WT $\omega-6$ mice $(p<0.05)$.

\section{Locomotor recovery (the SFI)}

To assess the degree of motor functional loss and subsequent recovery after sciatic nerve crush in fat- 1 compared with WT $\omega$ - 6 mice, the SFI was used. The SFI measures motor functional loss on a scale from 0 to -100 , where 0 corresponds to normal function and a value of -100 corresponds to complete loss of function. There was statistically no difference in the baseline SFI between the WT $\omega-6(-2.5)$ and fat-1 $(-3.8)$ groups. SFI was then measured $7 \mathrm{~d}$ after injury. There was a 25 -fold decrease in the SFI score for the WT $\omega-6$ group $(-60)$. This was significantly worse than the 12-fold decrease seen for the fat-1 group $(-45)$; however, even in the fat-1 group, locomotor recovery was still significantly impaired compared with baseline (Fig. 4).

\section{Histological analysis}

Changes in the DRG. The response to injury was assessed 1 week after injury in L4 and L5 DRGs using ATF-3 as a marker of neuronal injury. ATF-3 is normally expressed at very low levels in uninjured DRG, as can be seen from the contralateral noninjured side (ATF- 3 expression is found in $\leq 1 \%$ neurons). In injured groups, the ipsilateral DRG expressed significantly more ATF- 3 than the contralateral side. However, $\sim 15 \%$ of WT $\omega-6$ DRG expressed ATF-3, which was significantly higher than the $7 \%$ of DRGs expressing ATF-3 seen in fat- 1 mice (Fig. $5 A$ ). Immunostaining for GAP-43, a regeneration-associated protein, was low in uninjured DRG neurons in both WT $\omega-6$ and fat-1 mice (16\%). There was no significant difference in the percentage of GAP-43-immunoreactive neurons between the two groups. GAP-43 intensity was greatly increased $7 \mathrm{~d}$ after nerve crush in injured DRG neurons from both WT $\omega-6$ and fat- 1 mice. There was no statistical difference in the percentage of GAP-43-positive 
DRG neurons after nerve crush between WT $\omega-6(31 \%)$ and fat-1 (32\%) mice (Fig. 5B).

Changes in the sciatic nerve. To assess the recovery of nerves histologically, we used NF200 as an axonal marker. After injury, nerves die back through wallerian degeneration and then begin to regenerate. Examination of the NF200 labeling $7 \mathrm{~d}$ after injury showed that fat 1 transgenic mice had more NF200-labeled axons $6 \mathrm{~mm}$ rostral to the injury site compared with WT $\omega-6$ animals (Fig. 6A). Quantification confirmed that the percentage of area occupied by NF200 staining in the ipsilateral side compared with the non-injured contralateral side was significantly greater in the fat-1 mice (87\%) compared with WT $\omega-6$ mice $(61 \%)$. We then looked at the regeneration marker GAP-43 (Fig. 6B) in the sciatic nerve. GAP-43 staining was greatly upregulated in the nerves of both groups of animals after injury. However, no significant difference between the percentage area covered by GAP-43 staining between WT $\omega-6$ and fat-1 mice was found.

The response of muscle to $P N I$

Once denervated after a PNI, muscle will begin to atrophy. To assess the level of atrophy, wet muscle weight and average muscle fiber area were examined. Muscle weight was significantly protected in fat-1 muscle compared with WT muscle 1 week after injury, with only $19 \%$ reduction in muscle mass compared with $28 \%$ in WT (Fig. 7A). We confirmed this observation by looking at muscle fiber area and found that fiber area decreased significantly less in the fat-1 group (21\%) compared with the WT group (34\%) $(p<0.05)$ (Fig. $7 B)$.

\section{Discussion}

Our results suggest that a raised tissue level of $\omega$-3 PUFAs, through expression of the fat-1 gene, increases the resistance to nerve injury. Analysis of the fatty acid content of phospholipids demonstrated that the concentration of the C:22- $\omega-3$ PUFAs increased significantly, with a concomitant decrease in the concentration of C:20/22- $\omega$-6 PUFAs in both the DRG and spinal cord of mice expressing the fat-1 gene. Of particular interest, the lipid species showing the greatest change was DPAn-3. There have been many studies, both in vitro and in vivo, demonstrating the neuroprotective effects of DHA (Belayev et al., 2009; Ménard et al., 2009; Ward et al., 2010), but little work has been done on the role DPAn-3 can play in injury and disease (Kelly et al., 2011).

The ability to protect neurons against injury is crucial to successful recovery because regeneration can only occur from neurons that survive the initial trauma. DRGs cultured from fat-1 mice showed a robust resilience against both mechanical stretch and hypoxia compared with WT. In a model of SCI, $\omega-6$ PUFAs lead to a worsened outcome (King et al., 2006). No difference in the response to injury between WT and WT $\omega-6$ groups was observed in the present study, suggesting that increasing endogenous $\omega$-6 PUFAs does not exacerbate injury in vitro. It has been hypothesized that the deleterious effects of $\omega-6$ PUFAs after SCI are attributable to their effects on animals per group).

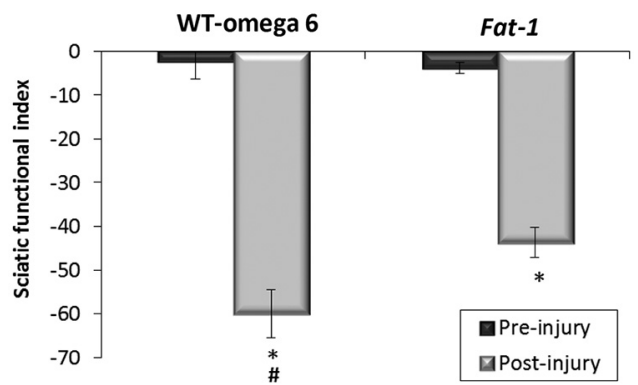

Figure 4. Motor function recovery after sciatic nerve crush assessed with the SFI. Functional motor recovery in mice was quantified by measuring the SFI. Print length (PL), toe spread (TS), and intermediary toe spread (ITS) were measured, and the SFI was calculated before injury and day 7 after injury. There was no significant difference between pre-injury values in the two groups. On day 7 after injury, there was a significant loss in activity $\left({ }^{*} p<0.05\right)$, although this loss was significantly less in the fat-1 group compared with WT $\omega-6$ mice ( $\left.{ }^{\#} p<0.05\right)$. Error bars indicate SEM for $n=6$ animals per group.

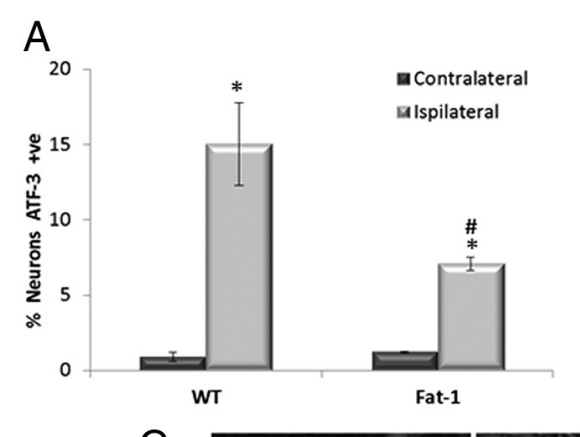

$\mathrm{B}$

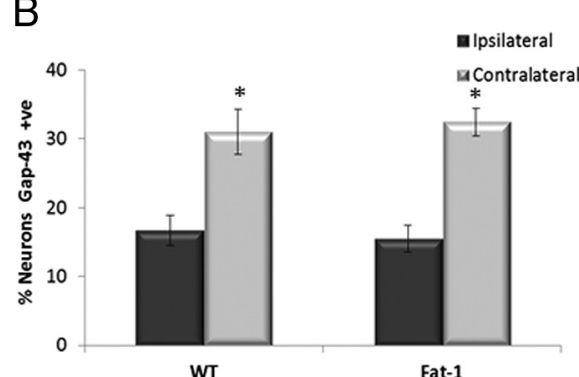

WT

Fat-1

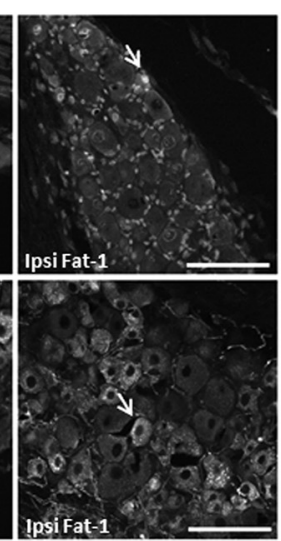

Figure 5. ATF-3 and GAP-43 expression in DRG neurons $7 \mathrm{~d}$ after sciatic nerve injury. Immunoreactivity for ATF-3 (A) and GAP-43 $(\boldsymbol{B})$ was analyzed in L4 and L5 DRG neurons removed $7 \mathrm{~d}$ after sciatic nerve crush. C, Example of neurons positive for ATF-3 and GAP-43 are marked with a yellow arrow. Scale bar, $100 \mu \mathrm{m}$. ATF-3 and GAP-43 staining detected in lumbar DRG neurons contralateral (Contra) to nerve injury was low. Both ATF-3 and GAP-43 staining was seen in a high proportion of cells in the ipsilateral (Ipsi) DRG after injury ( $\left.{ }^{*} p<0.05\right)$. There was a significant difference between WT $\omega-6$ and fat- 1 in the percentage of neurons expressing ATF-3 after injury, with a smaller percentage of ATF-3 neurons in fat- 1 mice ( $\left.{ }^{\#} p<0.05\right)$ (means \pm SEM, $n=11$

inflammation; therefore, the lack of an inflammatory component to the injury models used in this study could explain the contrasting results. Alternatively, the method of administration (expression of the fat-1 gene vs systemic injection after injury in vivo) could account for the differences.

In agreement with previous published findings (Calderon and Kim, 2004; Robson et al., 2010), the results in this study demonstrate the intrinsic neurotrophic properties of $\omega$-3 PUFAs in vitro, with DRGs from fat-1 mice showing more complex neurite outgrowth and an increased maximum neurite length compared with WT $\omega-6$. This increased outgrowth could be attributable to 

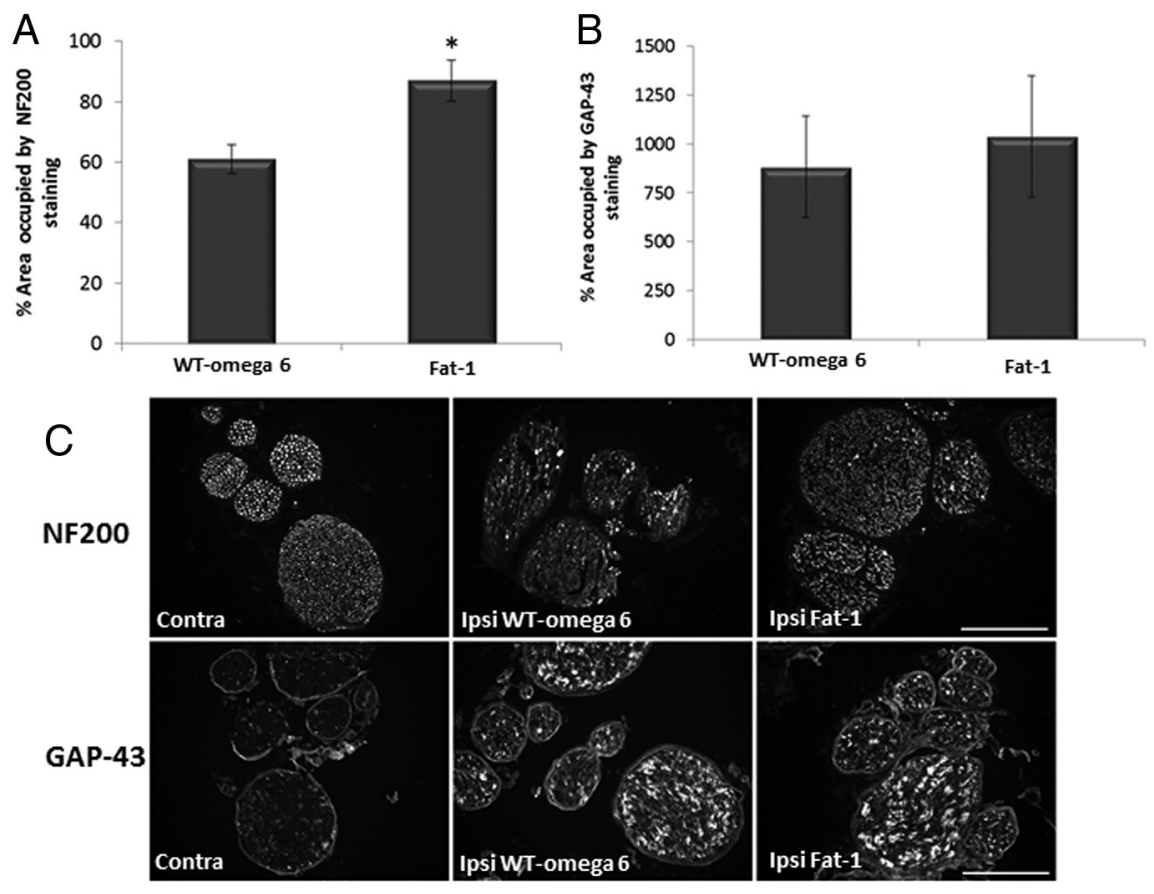

Figure 6. GAP-43 and NF200 staining after sciatic nerve injury. Immunoreactivity for GAP-43 (A) and NF200 (B) was analyzed in transverse sections of sciatic nerve, $6 \mathrm{~mm}$ from the crush site $7 \mathrm{~d}$ after injury. Scale bar, $100 \mu \mathrm{m}$. C, GAP-43 staining was significantly increased after injury. There was no statistical difference between fat- 1 and WT $\omega-6$ groups. NF200 staining decreased in both groups after injury, but there was significantly less staining in the WT $\omega$ - 6 group $\left({ }^{*} p<0.05\right.$, means \pm SEM, $n=11$ animals per group). Contra, Contralateral; Ipsi, ipsilateral.
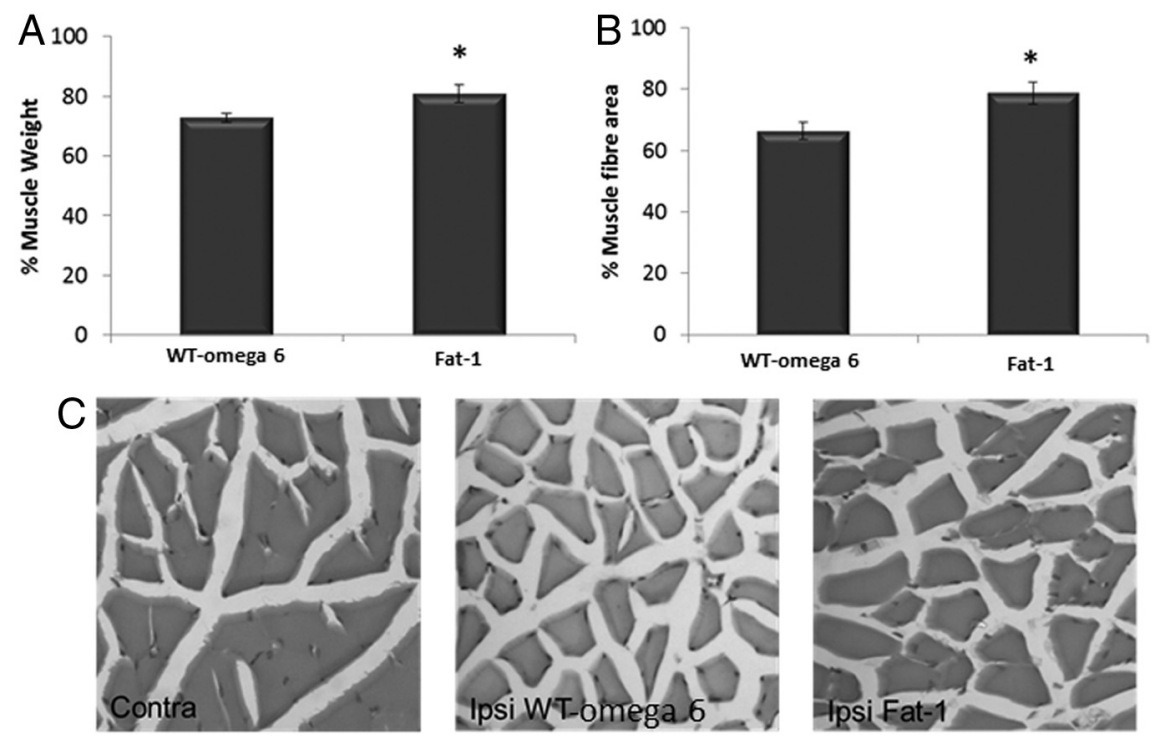

Figure 7. Response of the muscle after sciatic nerve injury. Muscle weight $(\boldsymbol{A})$ and muscle fiber area $(\boldsymbol{B})$ were analyzed $7 \mathrm{~d}$ after sciatic nerve crush. There was an increased muscle weight in the fat- 1 group compared with WT $\left({ }^{*} p<0.05\right)$. The muscle fiber area was also significantly higher compared with WT $\omega-6$ animals (means \pm SEM, $n=11$ animals per group). C, H\&E staining of contralateral (Contra) and ipsilateral (Ipsi) muscle from WT and fat-1 mice.

a reduced latency time required for neurons to begin to extend neurites, an increase in the rate of neurite outgrowth, or both.

The response to injury of mice expressing the fat-1 gene was then examined using the sciatic nerve crush model. Behavioral observations showed that endogenous $\omega$-3 PUFAs have a positive effect on the rate of sensory functional recovery. The SFI reflects locomotion and combines the coordination of motor and sensory reflexes. At $7 \mathrm{~d}$ after injury, there was a small but significant difference, with fat-1 mice regaining comparatively more function, thus indicative of an increased rate of recovery. The VF test is assessing the motor response to a sensory stimulus. As expected, both groups lost sensation after the crush injury. Sensation was beginning to recover over the period tested, and, by $4 \mathrm{~d}$ after crush, withdrawal threshold for the fat-1 mice was significantly lower than for WT $\omega-6$ mice. This trend continued up to day 7 when the animals were killed and could suggest that $\omega$-3 PUFAs increase rate of regeneration. However, by $1 \mathrm{~d}$ after injury, there was already a difference in the force required to induce a response between the two groups, although not yet statistically significant. This is a strong indication that the injury was less severe in the fat-1 mice, likely because of the neuroprotective properties of $\omega$-3 PUFA leading to more spared axons. This could be the explanation for the observed improvements in both the VF and the SFI assessments. Additionally, it could be hypothesized that $\omega-3$ PUFAs led to an increase in collateral sprouting from spared axons in the sciatic nerve, and this could further contribute to the increased rate of recovery seen in mice expressing the fat- 1 gene. The distances over which regeneration must sometimes occur in humans are much greater than in the mouse, and collateral sprouting in humans is limited compared with that seen in rodents (Healy et al., 1996; Rajan et al., 2003); therefore, a therapy that could protect nerves and enhance collateral sprouting may be beneficial.

Observations of functional recovery were also mapped to changes in neurochemical expression. ATF-3 is upregulated after injury (Tsujino et al., 2000; Averill et al., 2004) and downregulated after reinnervation of the peripheral targets (Seijffers et al., 2006). ATF-3 was significantly lower in DRGs harvested from fat-1 mice compared with WT $\omega-6$ animals. ATF-3 would be reduced if there was neuroprotection (i.e., fewer axons affected by the crush) or an increased reinnervation of targets. It should be noted, however, that ATF-3 levels can decline naturally even if reinnervation fails, and ATF-3 levels can be influenced by factors such as nerve growth factor expression (Averill et al., 2004; Kataoka et al., 2007 ), which could be increased by $\omega-3$ PUFAs. Compared with the WT $\omega-6$ group, there was more NF200 staining in the sciatic nerve $6 \mathrm{~mm}$ distal to the crush site in mice expressing the fat-1 gene, indicating that these mice had more axons at this point. It cannot be known from this whether these were spared axons, regenerating axons, or both. Nevertheless, it supports the conclusions from the behavioral data that fat-1 mice regain function at 
an earlier time point. GAP-43 is a regeneration-associated protein that is critical for successful regeneration and has been shown to be increased by $\omega$-3 PUFA in vitro (Cao et al., 2005). GAP-43 can be upregulated in both neurons and Schwann cells and, after the crush injury, was seen to be increased in the DRG and sciatic nerve. Interestingly, there was no difference in expression between WT $\omega-6$ and fat- 1 groups, suggesting that $\omega-3$ PUFAs do not enhance GAP-43 expression in vivo, at least in this model.

Our results also demonstrated some muscle protection in the fat-1 group. This could be a direct protection or an indirect effect (i.e., more spared axons or faster reinnervation of muscle would prevent atrophy). The muscle protection in the fat- 1 group might also equate with an increased muscle strength, which may lead to fat- 1 mice regaining the ability to perform the functional tests earlier. This is an interesting finding because, although myofibers are able to recover from denervation atrophy after the nervemuscle contacts have been reestablished, muscle function often fails to recover completely. This effect is amplified in humans in which muscle is often denervated for a longer period of time. $\omega$-3 PUFAs, such as eicosapentaenoic acid, have been implicated previously in muscle protection in other models, such as muscle dystrophy (Machado et al., 2011).

PUFAs are essential components of membrane phospholipids and have a specific influence on membrane properties. Membranes enriched in C:22 $\omega$-3 PUFAs demonstrate significant increases in membrane fluidity and lipid raft formation (for review, see Stillwell and Wassall, 2003) and can directly or indirectly affect the function of a number of membrane proteins, such as receptors (Yamashima, 2008; Lafourcade et al., 2011) and ion channels (Lauritzen et al., 2000; Danthi et al., 2005; Ye et al., 2010). $\omega$-3 PUFAs are also known to act as a ligand for the retinoid X receptor (RXR) and activate peroxisome proliferatoractivated receptors. Therefore, increasing endogenous $\omega$-3 PUFAs produces widespread effects on the expression of genes, and some of these could be responsible for the neuroprotective and neurotrophic effects seen in this study (Jump, 2002). For example, $\omega$-3 PUFAs and their metabolites have been shown to upregulate the expression of the Bcl-2 family of anti-apoptotic proteins but downregulate the apoptotic proteases caspase- 3 and -9 and pro-apoptotic signaling proteins in a number of cell types, including neurons (Lukiw et al., 2005; Bazan, 2009). It has also been suggested that $\omega$-3 PUFAs enhance expression of neurotrophins, including brain-derived neurotrophic factor, which can have both neuroprotective and pro-regenerative effects (Bousquet et al., 2009). It is important to note the observations made in a model of sciatic nerve crush in rat, in which the presence of RXR and retinoic acid receptor RAR was shown in regenerating nerves, in particular at $7 \mathrm{~d}$ after injury (Zhelyaznik and Mey, 2006), indicating that these receptors may have an important role in the regenerative response. Furthermore, a role of retinoid signaling (likely to by modulated by DHA) in the regeneration of sensory neurons in the spinal cord has also been demonstrated (Wong et al., 2006). Interestingly, in a model of corneal nerve regeneration after surgery, it has been shown that DHA has strong pro-regenerative effects in combination with pigment epithelial growth factor, and the authors hypothesized that this may involve formation of the DHA metabolite neuroprotectin D1 (Cortina et al., 2010).

In conclusion, both in vitro and in vivo experiments point to the neuroprotective and neurotrophic effects of endogenous $\omega$-3 PUFAs, which could lead to a secondary effect on regeneration after a PNI. However, the time points used in the present PNI experiments, similar to those reported by others with agents such as erythropoietin (Elfar et al., 2008), make it difficult to distinguish between neuroprotection and an increase in regeneration, and additional research into the mechanisms leading to the increased rate of recovery is warranted, in particular by monitoring the animals over a longer time period after injury.

\section{References}

Averill S, Michael GJ, Shortland PJ, Leavesley RC, King VR, Bradbury EJ, McMahon SB, Priestley JV (2004) NGF and GDNF ameliorate the increase in ATF3 expression which occurs in dorsal root ganglion cells in response to peripheral nerve injury. Eur J Neurosci 19:1437-1445.

Bazan NG (2009) Cellular and molecular events mediated by docosahexaenoic acid-derived neuroprotectin D1 signaling in photoreceptor cell survival and brain protection. Prostaglandins Leukot Essent Fatty Acids 81:205-211.

Belayev L, Khoutorova L, Atkins KD, Bazan NG (2009) Robust docosahexaenoic acid-mediated neuroprotection in a rat model of transient, focal cerebral ischemia. Stroke 40:3121-3126.

Bousquet M, Gibrat C, Saint-Pierre M, Julien C, Calon F, Cicchetti F (2009) Modulation of brain-derived neurotrophic factor as a potential neuroprotective mechanism of action of omega-3 fatty acids in a parkinsonian animal model. Prog Neuropsychopharmacol Biol Psychiatry 33:1401-1408.

Calderon F, Kim HY (2004) Docosahexaenoic acid promotes neurite growth in hippocampal neurons. J Neurochem 90:979-988.

Cao D, Xue R, Xu J, Liu Z (2005) Effects of docosahexaenoic acid on the survival and neurite outgrowth of rat cortical neurons in primary cultures. J Nutr Biochem 16:538-546.

Cortina MS, He J, Li N, Bazan NG, Bazan HE (2010) Neuroprotectin D1 synthesis and corneal nerve regeneration after experimental surgery and treatment with PEDF plus DHA. Invest Ophthalmol Vis Sci 51:804-810.

Danthi SJ, Enyeart JA, Enyeart JJ (2005) Modulation of native T-type calcium channels by omega-3 fatty acids. Biochem Biophys Res Commun 327:485-493

Dyall SC, Michael-Titus AT (2008) Neurological benefits of omega-3 fatty acids. Neuromolecular Med 10:219-235.

Dyall SC, Michael GJ, Whelpton R, Scott AG, Michael-Titus AT (2007) Dietary enrichment with omega- 3 polyunsaturated fatty acids reverses agerelated decreases in the GluR2 and NR2B glutamate receptor subunits in rat forebrain. Neurobiol Aging 28:424-439.

Elfar JC, Jacobson JA, Puzas JE, Rosier RN, Zuscik MJ (2008) Erythropoietin accelerates functional recovery after peripheral nerve injury. J Bone Joint Surg Am 90:1644-1653.

Folch J, Lees M, Sloane Stanley GH (1957) A simple method for the isolation and purification of total lipid from animal tissues. J Biol Chem 226:497-509.

Gladman SJ, Ward RE, Michael-Titus AT, Knight MM, Priestley JV (2010) The effect of mechanical strain or hypoxia on cell death in subpopulations of rat dorsal root ganglion neurons in vitro. Neuroscience 171:577-587.

Healy C, LeQuesne PM, Lynn B (1996) Collateral sprouting of cutaneous nerves in man. Brain 119:2063-2072.

Huang WL, King VR, Curran OE, Dyall SC, Ward RE, Lal N, Priestley JV, Michael-Titus AT (2007) A combination of intravenous and dietary docosahexaenoic acid significantly improves outcome after spinal cord injury. Brain 130:3004-3019.

Inserra MM, Bloch DA, Terris DJ (1998) Functional indices for sciatic, peroneal, and posterior tibial nerve lesions in the mouse. Microsurgery 18:119-124.

Jaquet JB, Luijsterburg AJ, Kalmijn S, Kuypers PD, Hofman A, Hovius SE (2001) Median, ulnar, and combined median-ulnar nerve injuries: functional outcome and return to productivity. J Trauma 51:687-692.

Jump DB (2002) Dietary polyunsaturated fatty acids and regulation of gene transcription. Curr Opin Lipidol 13:155-164.

Kang JX, Wang J, Wu L, Kang ZB (2004) Transgenic mice: fat-1 mice convert n-6 to n-3 fatty acids. Nature 427:504.

Kataoka K, Kanje M, Dahlin LB (2007) Induction of activating transcription factor 3 after different sciatic nerve injuries in adult rats. Scand J Plast Reconstr Surg Hand Surg 41:158-166.

Kelly L, Grehan B, Chiesa AD, O’Mara SM, Downer E, Sahyoun G, Massey KA, Nicolaou A, Lynch MA (2011) The polyunsaturated fatty acids, EPA and DPA exert a protective effect in the hippocampus of the aged rat. Neurobiol Aging 32:2318.e1-e15. 
King VR, Huang WL, Dyall SC, Curran OE, Priestley JV, Michael-Titus AT (2006) Omega-3 fatty acids improve recovery, whereas omega-6 fatty acids worsen outcome, after spinal cord injury in the adult rat. J Neurosci 26:4672-4680.

Lafourcade M, Larrieu T, Mato S, Duffaud A, Sepers M, Matias I, De SmedtPeyrusse V, Labrousse VF, Bretillon L, Matute C, Rodríguez-Puertas R, Lay é S, Manzoni OJ (2011) Nutritional omega-3 deficiency abolishes endocannabinoid-mediated neuronal functions. Nat Neurosci 14:345-350.

Lauritzen I, Blondeau N, Heurteaux C, Widmann C, Romey G, Lazdunski M (2000) Polyunsaturated fatty acids are potent neuroprotectors. EMBO J 19:1784-1793.

Lukiw WJ, Cui JG, Marcheselli VL, Bodker M, Botkjaer A, Gotlinger K, Serhan CN, Bazan NG (2005) A role for docosahexaenoic acid-derived neuroprotectin D1 in neural cell survival and Alzheimer disease. J Clin Invest 115:2774-2783.

Machado RV, Mauricio AF, Taniguti AP, Ferretti R, Neto HS, Marques MJ (2011) Eicosapentaenoic acid decreases TNF-alpha and protects dystrophic muscles of $\mathrm{mdx}$ mice from degeneration. J Neuroimmunol 232:145-150.

Makwana M, Dyall S, Raivich G, Michael-Titus A (2006) Effect of docosahexaenoic acid, an omega-3 polyunsaturated fatty acid, in a mouse facial nerve injury model. Br Pharmacol Soc 4:18IP. http://www.pa2online.org/ abstracts/Vol4Issue2abst181P.pdf.

Manku MS, Horrobin DF, Huang YS, Morse N (1983) Fatty acids in plasma and red cell membranes in normal humans. Lipids 18:906-908.

Ménard C, Patenaude C, Gagn é AM, Massicotte G (2009) AMPA receptormediated cell death is reduced by docosahexaenoic acid but not by eicosapentaenoic acid in area CA1 of hippocampal slice cultures. J Neurosci Res 87:876-886.

Morrison WR, Smith LM (1964) Preparation of fatty acid methyl esters and dimethylacetals from lipids with boron trifluoride-methanol. J Lipid Res 5:600-608.

Rajan B, Polydefkis M, Hauer P, Griffin JW, McArthur JC (2003) Epidermal reinnervation after intracutaneous axotomy in man. J Comp Neurol 457:24-36.

Robson LG, Dyall S, Sidloff D, Michael-Titus AT (2010) Omega-3 polyunsaturated fatty acids increase the neurite outgrowth of rat sensory neurones throughout development and in aged animals. Neurobiol Aging 31:678-687.
Rosberg HE, Carlsson KS, Höjgård S, Lindgren B, Lundborg G, Dahlin LB (2005) Injury to the human median and ulnar nerves in the forearmanalysis of costs for treatment and rehabilitation of 69 patients in southern Sweden. J Hand Surg Br 30:35-39.

Seijffers R, Allchorne AJ, Woolf CJ (2006) The transcription factor ATF-3 promotes neurite outgrowth. Mol Cell Neurosci 32:143-154.

Simopoulos AP (2006) Evolutionary aspects of diet, the omega-6/omega-3 ratio and genetic variation: nutritional implications for chronic diseases. Biomed Pharmacother 60:502-507.

Simopoulos AP (2008) The importance of the omega-6/omega-3 fatty acid ratio in cardiovascular disease and other chronic diseases. Exp Biol Med (Maywood) 233:674-688.

Stillwell W, Wassall SR (2003) Docosahexaenoic acid: membrane properties of a unique fatty acid. Chem Phys Lipids 126:1-27.

Tsujino H, Kondo E, Fukuoka T, Dai Y, Tokunaga A, Miki K, Yonenobu K, Ochi T, Noguchi K (2000) Activating transcription factor 3 (ATF3) induction by axotomy in sensory and motoneurons: a novel neuronal marker of nerve injury. Mol Cell Neurosci 15:170-182.

Varejão AS, Meek MF, Ferreira AJ, Patrício JA, Cabrita AM (2001) Functional evaluation of peripheral nerve regeneration in the rat: walking track analysis. J Neurosci Methods 108:1-9.

Ward RE, Huang W, Curran OE, Priestley JV, Michael-Titus AT (2010) Docosahexaenoic acid prevents white matter damage after spinal cord injury. J Neurotrauma 27:1769-1780.

Wong LF, Yip PK, Battaglia A, Grist J, Corcoran J, Maden M, Azzouz M, Kingsman SM, Kingsman AJ, Mazarakis ND, McMahon SB (2006) Retinoic acid receptor beta 2 promotes functional regeneration of sensory axons in the spinal cord. Nat Neurosci 9:243-250.

Yamashima T (2008) A putative link of PUFA, GPR40 and adult-born hippocampal neurons for memory. Prog Neurobiol 84:105-115.

Ye S, Tan L, Ma J, Shi Q, Li J (2010) Polyunsaturated docosahexaenoic acid suppresses oxidative stress induced endothelial cell calcium influx by altering lipid composition in membrane caveolar rafts. Prostaglandins Leukot Essent Fatty Acids 83:37-43.

Zhelyaznik N, Mey J (2006) Regulation of retinoic acid receptors alpha, beta and retinoid $\mathrm{X}$ receptor alpha after sciatic nerve injury. Neuroscience 141:1761-1774 